\title{
Palatability mapping: a koala's eye view of spatial variation in habitat quality
}

\author{
Ben D. Moore, ${ }^{1,4}$ Ivan R. Lawler, ${ }^{2,5}$ Ian R. Wallis, ${ }^{1}$ Colin M. Beale, ${ }^{3,6}$ and William J. Foley ${ }^{1}$ \\ ${ }^{1}$ Evolution, Ecology and Genetics, Research School of Biology, Australian National University, Canberra, ACT 0200 Australia \\ ${ }^{2}$ School of Earth and Environmental Sciences, James Cook University of North Queensland, Douglas, Queensland 4811 Australia \\ ${ }^{3}$ Ecology Group, Macaulay Land Use Research Institute, Aberdeen AB158QH United Kingdom
}

\begin{abstract}
Ecologists trying to understand the value of habitat to animals must first describe the value of resources contained in the habitat to animals and, second, they must describe spatial variation in resource quality at a resolution relevant to individual animal foraging. We addressed these issues in a study of koalas (Phascolarctos cinereus) in a Eucalyptus woodland. We measured beneficial and deterrent chemical characteristics as well as the palatability of trees using a near-infrared spectroscopic model based on direct feeding experiments. Tree use by koalas was influenced by tree size and foliar quality but was also context-dependent: trees were more likely to be visited if they were surrounded by small, unpalatable trees or by large, palatable trees. Spatial autocorrelation analysis and several mapping approaches demonstrated that foliar quality is spatially structured in the woodland at a scale relevant to foraging decisions by koalas and that the spatial structure is an important component of habitat quality.
\end{abstract}

Key words: associational defense; Australian Eucalyptus woodland; formylated phloroglucinol compounds; generalized additive mixed modeling; habitat quality; koala; NIRS; patch use; Phascolarctos cinereus; plant secondary metabolites; spatial autocorrelation.

\section{INTRODUCTION}

A fundamental aspect of animal habitat quality is the quality and amount of food available. To understand this, ecologists need to be able to describe variation in food quality at scales that are relevant to foraging individuals through to scales that are relevant to populations and species (Searle et al. 2007). A concept closely linked to food quality is palatability, an emergent property resulting from a complex series of interactions. Palatability must be deduced from herbivores' feeding behavior, which is a product of the herbivores' current nutritional state, their past experiences (if any) with the food, and their perceptions of the trade-off between any detrimental qualities of the food (e.g., plant secondary metabolites, PSMs) against the food's positive nutritional qualities (Bryant et al. 1991, Provenza et al. 1998). Feeding studies with captive animals can often reveal animals' preferences from among a limited range of foods (Hjältén et al. 2004, Schreiber and Swihart 2009), or identify factors such as PSMs that cause them to

Manuscript received 18 September 2009; revised 11 February 2010; accepted 3 March 2010; final version received 25 March 2010. Corresponding Editor: M. D. Dearing.

${ }^{4}$ Present address: Ecology Group, Macaulay Land Use Research Institute, Craigiebuckler, Aberdeen AB158QH United Kingdom. E-mail: b.moore@macaulay.ac.uk

${ }^{5}$ Present address: Department of the Environment, Water, Heritage and the Arts, GPO Box 787, Canberra, ACT 2601 Australia.

${ }^{6}$ Present address: Department of Biology (Area 18), P.O. Box 373, University of York, YO10 5YW United Kingdom. reject some foods (Bryant et al. 1983, Lawler et al. 1999), but they are not necessarily useful for predicting foraging behavior by wild animals that are faced with more complex choices and trade-offs while foraging in heterogeneous environments.

The defensive and nutritional chemistry of many, if not most, plant populations is highly variable (Lawler et al. 2000) and this variation is often spatially structured (Covelo and Gallardo 2004, Andrew et al. 2007). Spatial hotspots of highly palatable or highly unpalatable plants are ecologically important, because the likelihood of a plant being eaten is a product not only of its own characteristics but also of the context in which it occurs (Feng et al. 2009). Plants experience associational defense or associational resistance when they benefit from reduced herbivory because of their association with neighboring plants, and associational susceptibility if the association is detrimental (Atsatt and O'Dowd 1976, Barbosa et al. 2009). A plant can experience associational resistance from unpalatable neighboring plants when herbivores make foraging decisions at the scale of a patch of plants, or by association with palatable neighbors if they act as attractant-decoys when herbivores forage at an individual-plant scale (Bergvall et al. 2006). Whether such associational effects occur, and whether spatial patches of trees of similar palatability are of any significance to foraging herbivores thus depends upon the scale of the herbivore's foraging movements (Milchunas and Noy-Meir 2002). A recent meta-analysis shows that although both associational resistance and susceptibility occur in plant-mammal 


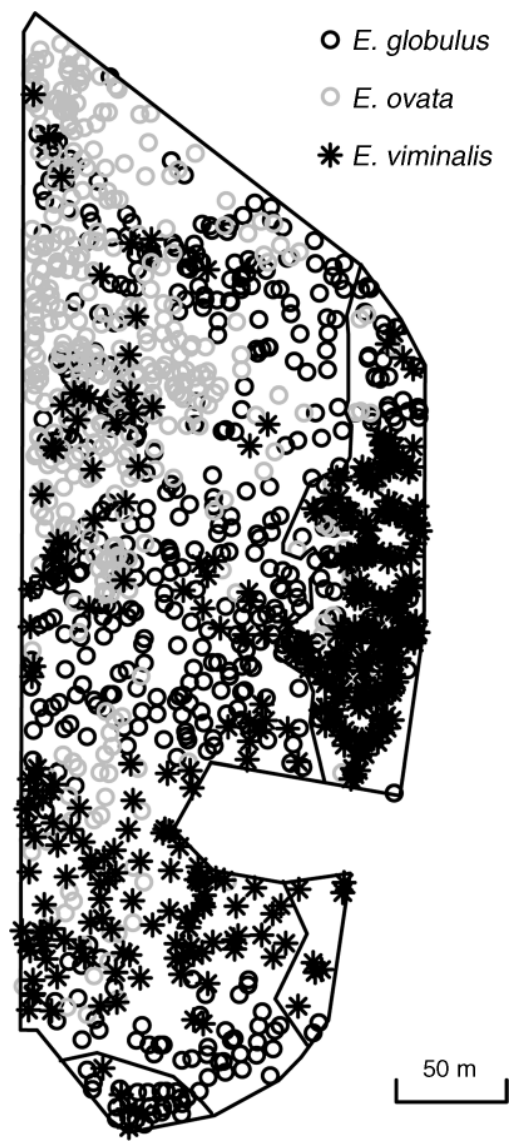

FIG. 1. Spatial distribution of Eucalyptus globulus, E. ovata, and E. viminalis trees in the study area at the 7.6-ha fenced Koala Conservation Centre on Phillip Island, Victoria, Australia. The fenced area is divided into four regions, indicated by solid lines. The largest region contains only remnant trees, and the three smaller areas on the lower and right-hand edges contain only planted trees.

interactions, the former is more likely (Barbosa et al. 2009). Few studies have looked for these effects in continuous, natural plant associations, because the environment has seldom been described at the spatial resolution required. However, any discussion of spatial context in plant-herbivore interactions should explicitly consider heterogeneity of plant associations.

In this study, we aimed to comprehensively describe the spatial distribution of foliar chemistry and palatability in a patch of Phascolarctos cinereus Goldfuss (koala) habitat large enough to include multiple koala home ranges. The koala (see Plate 1) is a highly specialized arboreal folivore that feeds almost exclusively on Eucalyptus L'Hérit. species (Martin and Handasyde 1999). Therefore, individual trees represent an unambiguous and discrete unit of habitat selection. Describing the habitat in such detail makes possible the investigation of spatial dependence in foliar palatability and an assessment of whether spatial dependence and spatial context influence the use of trees by koalas.
To achieve this goal, we used near-infrared reflectance spectroscopy (NIRS) (Foley et al. 1998). In NIRS, statistical calibrations are established between nearinfrared spectra and qualitative or quantitative attributes of organic samples such as tree leaves. Because it is correlative, NIRS is ideal for predicting emergent properties of plant material, such as palatability or digestibility of foods (McIlwee et al. 2001, DeGabriel et al. 2008) or rates of decomposition (Stolter et al. 2006), and it does not require a priori knowledge of the underlying chemistry. In this study, we used NIRS to develop an empirical calibration for palatability of foliage to koalas (i.e., voluntary dry matter intake of Eucalyptus foliage).

Tree use and diet choice by koalas have previously been linked, with various degrees of support, to tree size and species and to foliar concentrations of nitrogen, phosphorous, potassium, fiber, volatile terpenes, soluble sugars, and leaf moisture (Moore and Foley 2000). However, the single most powerful deterrent of koala feeding is a group of lipid-soluble phenolics known as the formylated phloroglucinol compounds, or FPCs (Moore and Foley 2005, Moore et al. 2005). Thus, one testable hypothesis arising from measurement of foliage palatability was that the NIRS predictions of palatability were negatively related to concentrations of FPCs and positively related to concentrations of nitrogen. NIRS calibrations for palatability have been developed previously for greater gliders (Petauroides volans Kerr) and common ringtail possums (Pseudocheirus peregrinus Ogilby) feeding on Eucalyptus (McIlwee et al. 2001, Wallis and Foley 2003), but they have not been applied to studies of wild animals. A second hypothesis was that the use of trees by wild koalas can be predicted as well as or better by these NIRS calibrations than by combinations of individually measured chemical constituents.

\section{Methods}

\section{Study site and species}

The study site was the Koala Conservation Centre, a 7.6-ha fenced koala reserve on Phillip Island $\left(38^{\circ} 28^{\prime} \mathrm{S}\right.$, $145^{\circ} 13^{\prime}$ E), Victoria, Australia. Approximately 20 koalas foraged freely throughout the reserve, which included 5.9 ha of remnant woodland dominated by the trees Eucalyptus globulus Labill. and E. ovata Labill. and to a lesser extent by $E$. viminalis Labill. The eastern side of the reserve was formerly cleared pasture land that had been replanted with $\sim 400$ trees, which averaged $6 \mathrm{~m}$ in height at the time of this study (Fig. 1).

All trees in the reserve were individually marked and mapped before this study commenced. In January 1997, diameters at breast height $(130 \mathrm{~cm})$, or $\mathrm{dbh}$, were measured and foliage samples were collected $(\sim 50 \mathrm{~g}$ wet mass) from all eucalypt trees, for a total of 376 remnant and 119 planted E. globulus, 383 remnant and 30 planted E. ovata, and 161 remnant and 215 planted E. viminalis. Samples of clean, fully expanded, adult foliage were 
sealed in plastic bags and immediately frozen. Samples were subsequently freeze-dried and then ground to pass a 1-mm screen using a Tecator cyclone mill (Tecator, North Ryde, NSW, Australia) prior to collection of NIR spectra or other chemical analysis.

\section{Measurements of foliar palatability}

Feeding measurements were made with three different groups of six individually housed koalas in three separate periods: April-July 1998, January-February 2002, and March 2003. On each occasion, six male koalas $(5.1-11.25 \mathrm{~kg})$ were captured from trees on French Island, Victoria, using standard techniques. In total, we determined the palatability of foliage from 50 E. globulus, 29 E. ovata, and 69 E. viminalis trees. Some trees were growing in the reserve, but many were collected from within a $50 \mathrm{~km}$ radius of it, on Phillip Island, French Island, and the mainland. The index of foliar palatability was the mean dry mass of foliage voluntarily eaten overnight by each of four male koalas, randomly assigned from a group of six koalas, under the no-choice feeding protocol (described by Moore et al. 2005). Briefly, at least $3 \mathrm{~kg}$ (fresh mass, including branches) of foliage from a single tree was weighed and presented to an individual koala overnight (from 17:00 to $08: 00$ hours). Uneaten and dropped foliage was reweighed the following morning, and the amount eaten was calculated and corrected for evaporative loss determined from a control branch placed outside the cage overnight. For each measurement, a sample of the foliage fed to koalas was frozen for subsequent collection of NIR spectra, other chemical analyses, and determination of dry mass.

\section{Development of NIRS calibration models}

For all dried and ground foliage samples, we collected the reflectance spectrum from 400 to $2500 \mathrm{~nm}$ using a NIRSystems 6500 scanning spectrophotometer with spinning cup attachment (NIRSystems, Silver Spring, Maryland, USA). Each sample was scanned twice or until the root mean square of two scans (stored as $\log (1 /$ reflectance)) was less than $3.0 \times 10^{-4}$, and the two spectra were averaged.

In most cases, mathematical transformations, one or both of the standard normal variate (SNV) and detrend transformations (Barnes et al. 1989), were applied to raw NIR spectra to reduce the influence of particle size. Predictive equations were then developed using modified partial least-squares regression (MPLS), with crossvalidation to prevent overfitting of models (Shenk and Westerhaus 1991). These regressions were performed on the first or second derivatives of the transformed spectra, using the software WinISI II, Version 1.02 (Infrasoft International, Port Matilda, Pennsylvania, USA). The optimum mathematical treatment was selected by minimizing the standard error of crossvalidation. Attempts were made to develop a universal NIRS calibration for palatability that could be applied across all three eucalypt species, in addition to combined two-species calibrations and individual single-species calibrations. To identify the best calibration, different combinations of data from each of the three experimental periods were tested.

Calibration data for total FPC concentrations were determined using the HPLC assay described by Moore et al. (2004b) and nitrogen was determined using a semimicro Kjeldahl technique. The NIRS calibrations used to measure these constituents are described in Moore and Foley (2005).

\section{Koala tree use data}

A community volunteer group, assisted by nature park rangers, systematically searched the reserve for at least three daylight hours once per month and recorded the tag numbers of all trees occupied by koalas. Between January 1993 and March 2004, 1522 such observations were recorded from Eucalyptus trees.

\section{Statistical modeling of tree use by koalas}

Generalized additive models (GAM) and generalized additive mixed models (GAMM) were used to describe koala visits to trees in the reserve. GAMs allow the consideration of multiple smooth explanatory variables, with the extension to GAMMs allowing spatially explicit models to be fitted, reducing the incidence of Type I statistical errors in spatial data sets (Lennon 2000). However, when the data set is restricted to individual tree species, the spatial structure of the tree use by koalas is not completely described and estimation of the spatial effects becomes inaccurate. Consequently, nonspatial GAMs were used to build single-species models and were then combined for all species using a spatially explicit GAMM. All GAM and GAMM models were implemented using the R package "mgcv" (Wood 2006, 2008).

Modeling was carried out in three stages. Firstly, pairs of nonspatial GAM models, with Poisson errors and a log-link were fitted to koala data for individual tree species, with tree size ( $\mathrm{dbh}$ ) and either palatability or, for comparison, total FPC and nitrogen content, as covariates. All covariates were smoothed with penalized regression splines with the maximum dimension fixed at 4. For each of the pair of models for each species, Akaike's information criterion (AIC) was used to select a minimum adequate model.

The second stage of the analysis combined all species in a spatially explicit GAMM model, again with Poisson errors and a log-link, and including an exponential covariance model of the residual spatial autocorrelation. Covariates were species identity, nitrogen, a species $\times$ nitrogen interaction, and smoothed $\mathrm{dbh}$ and FPC concentration. Predictions of individual tree use were generated and used to determine an index of neighborhood quality for use in the third stage of analysis. The neighborhood quality was the mean predicted number 


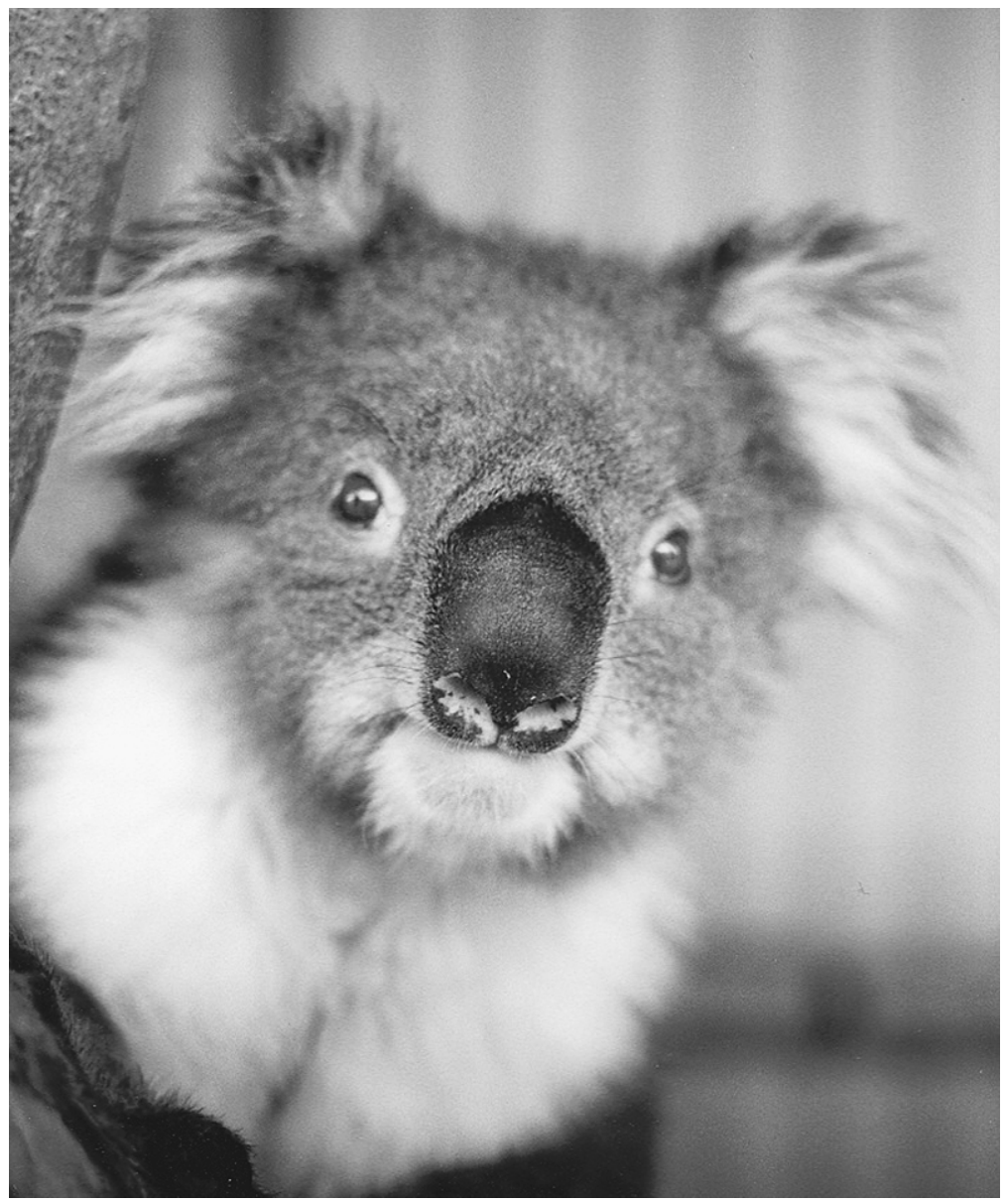

Plate 1. The koala is a highly specialized folivore, feeding almost exclusively on Eucalyptus foliage, but showing strong selectivity among individual trees, both between and within tree species. Photo credit: B. D. Moore.

of koala visits to all trees within a $25 \mathrm{~m}$ radius away from the focal tree.

The final stage of analysis was to repeat the first and second stages, but now including an additional smooth term describing the neighborhood quality estimated in the second stage. Goodness of fit was assessed using residual plots, and as there was some evidence of zeroinflation in the GAM models, the analysis was repeated using constrained zero-inflated generalized additive models (COZIGAM; Liu and Chan 2008) with the zero-inflation a function of tree size. These models showed improved fit but regression coefficients were essentially similar and consequently those models are not reported further.

\section{Spatial analysis of foliar characteristics}

The strength and scale of spatial autocorrelation in FPC concentrations and predicted DMI (dry matter intake) were determined by calculating Moran's $I$, an index of global spatial autocorrelation, at $10 \mathrm{~m}$ distance intervals from $10 \mathrm{~m}$ to $200 \mathrm{~m}$. Values of $I$ were tested for significance against 1000 permutations, and autocorrelo- grams were constructed with these data. These analyses excluded planted trees, because the aim was to understand spatial dependence in a natural woodland.

\section{Mapping}

A number of interpolated surfaces were produced to describe the phenotypic landscape within the reserve. This approach aimed to visualize spatial trends in foliar attributes of a single tree species, without regard for the size of individual trees or their contribution to the nutritional landscape facing herbivores. For this approach, the intensity $\hat{\lambda}_{\tau}(z)$ of the attribute of interest at a location $z$ in the study area A was determined by

$$
\hat{\lambda}_{\tau}(z)=\frac{\sum_{i=1}^{n} \frac{y_{i}}{\left(z-z_{i}\right)^{b}}}{\sum_{i=1}^{n} \frac{1}{\left(z-z_{i}\right)^{b}}} \quad z \in A
$$

where $z_{i}(i=1, \ldots, n)$ are locations of $n$ observed trees in the circular neighborhood with radius $\tau,\left(z-z_{i}\right)$ is the Euclidean distance between $z$ and $z_{i}$ in meters, $y_{i}$ is the 


\section{E. globulus}
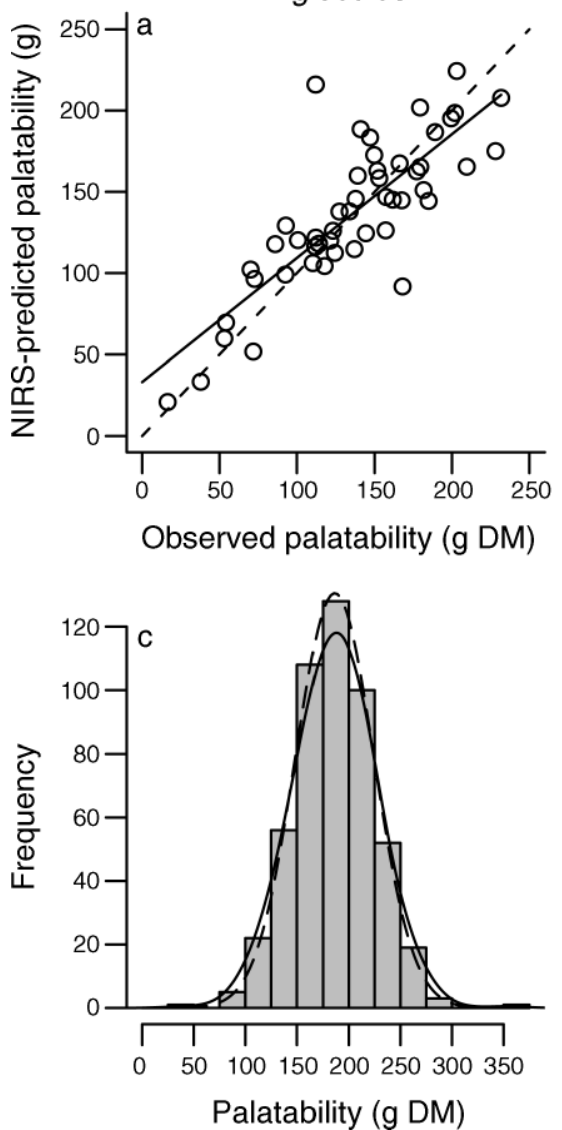

E. viminalis
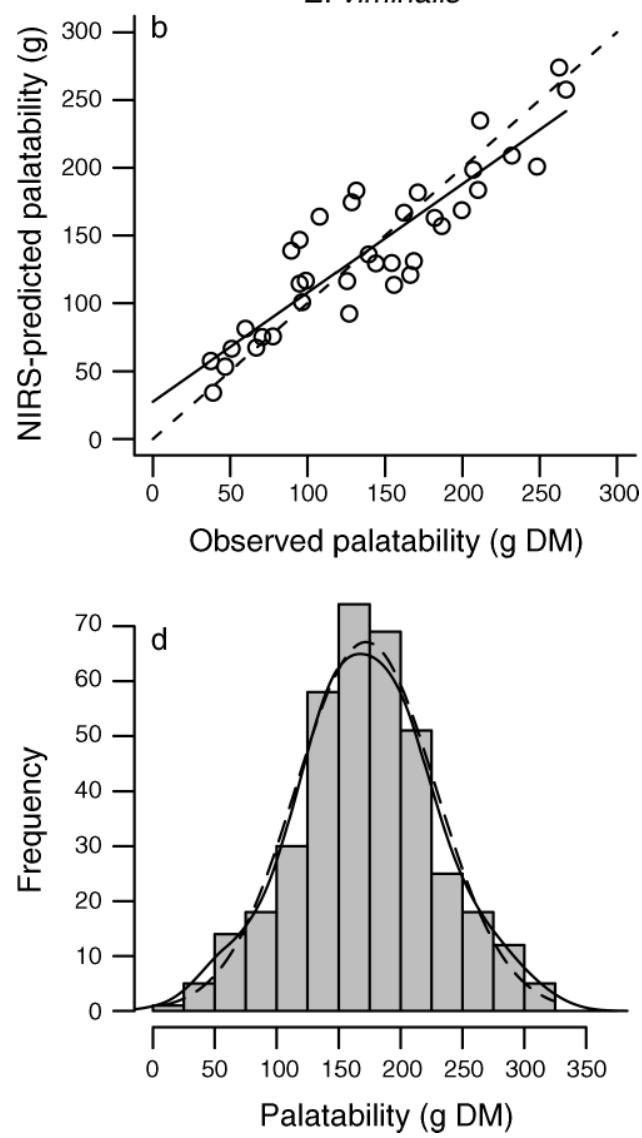

FIG. 2. Palatability of foliage to koalas (dry matter intake, in grams), predicted by near-infrared spectroscopic (NIRS) calibrations plotted against mean observed palatability to captive koalas $(n=4$ independent observations of feeding by koalas on each tree's foliage) for (a) E. globulus and (b) E. viminalis. Solid lines indicate ordinary least-squares linear regressions, and dashed lines show a 1:1 relationship. Panels (c) and (d) are histograms showing the distributions of NIRS-predicted palatability of $E$. globulus ( $n=495$ trees) and E. viminalis ( $n=380$ trees). Solid lines indicate scaled kernel density estimates, and dashed lines indicate scaled normal distributions.

attribute value at $z_{i}$, and $b$ is a positive real number called the power constant. Interpolated surfaces were also produced to describe the density of predicted (extracted from the final GAMM using the "predict" function in R) and observed koala visits throughout the reserve using

$$
\hat{\lambda}_{\tau}(z)=\frac{1}{p_{\tau}(z)}\left\{\sum_{i=1}^{n} k\left(z-z_{i}\right) y_{i}\right\} \quad z \in A
$$

The kernel, or probability density function, $k$, used to weight observations within the search radius $\tau$ was determined from 332 observations of movements by radio-collared female koalas from one tree to another within the reserve. For records to qualify, koalas must have remained under continuous direct visual observation from the moment they left the canopy of one tree until they entered another. These observations were made as part of another study (K. J. Marsh and B. D. Moore, unpublished data). The term $p_{\tau}(z)$ is an edge correction equivalent to the volume under the scaled kernel centered on $z$, which lies inside study area A.

\section{Results}

\section{NIRS predictions of foliar attributes}

The mean amount of foliage (dry matter, DM) eaten by koalas varied considerably among individual trees of Eucalyptus globulus $(16-232 \mathrm{~g} \mathrm{DM}$, mean $=136.6)$ and E. viminalis (37-267 g DM, mean $=139.2)$, but less so among E. ovata trees $(134-213 \mathrm{~g} \mathrm{DM}$, mean $=145)$. Mathematical treatment of spectra and descriptive statistics for NIRS calibrations of foliage palatability are described in the Appendix. The models for $E$. globulus and E. viminalis both had $r^{2}$ values of 0.78 and had standard errors of cross-validation of 33.2 and 39.4, respectively. NIRS-predicted palatability values agreed well with observed values and palatability was normally distributed for both species (Fig. 2). All attempts to develop a calibration encompassing more than one 
TABLE 1. Generalized additive models (GAM 1-5) separately describing koala visits to three Eucalyptus species at Phillip Island, Australia, and a generalized additive mixed model (GAMM 1) describing visits to all three species.

\begin{tabular}{|c|c|c|c|c|c|c|}
\hline Model and terms & Estimate & $\begin{array}{c}z \text { or } F \\
\text { smooth terms } \dagger\end{array}$ & $\begin{array}{c}\chi^{2} \text { or } t \\
\text { parametric terms }\end{array}$ & $P$ & $r^{2}$ & AIC \\
\hline $\begin{array}{l}\text { GAM 1: E. globulus } \\
\text { s(dbh) } \\
\text { s(Neighborhood) } \\
\text { s(FPC) } \\
\text { s(Nitrogen) }\end{array}$ & & $\begin{array}{r}489.124 \\
41.603 \\
13.805 \\
9.925\end{array}$ & & $\begin{array}{r}<0.001 \\
<0.001 \\
0.002 \\
0.022\end{array}$ & 0.32 & 1785.0 \\
\hline $\begin{array}{l}\text { GAM 2: E. globulus } \\
\text { s(dbh) } \\
\text { s(Neighborhood) } \\
\text { s(Palatability) }\end{array}$ & & $\begin{array}{r}535.6 \\
55.9 \\
20.0\end{array}$ & & $\begin{array}{l}<0.001 \\
<0.001 \\
<0.001\end{array}$ & 0.33 & 1785.1 \\
\hline $\begin{array}{l}\text { GAM 3: E. viminalis } \\
\text { s(dbh) } \\
\text { s(Neighborhood) } \\
\text { s(FPC) }\end{array}$ & & $\begin{array}{l}92.5 \\
55.5 \\
13.7\end{array}$ & & $\begin{array}{r}<0.001 \\
<0.001 \\
0.003\end{array}$ & 0.36 & 1101.5 \\
\hline $\begin{array}{l}\text { GAM 4: E. viminalis } \\
\text { s(dbh) } \\
\text { s(Neighborhood) } \\
\text { s(Palatability) }\end{array}$ & & $\begin{array}{r}106.7 \\
48.9 \\
16.7\end{array}$ & & $\begin{array}{l}<0.001 \\
<0.001 \\
<0.001\end{array}$ & 0.35 & 1102.8 \\
\hline $\begin{array}{l}\text { GAM 5: E. ovata } \\
\text { s(dbh) } \\
\text { Nitrogen }\end{array}$ & -0.12 & 203.9 & -3.2 & $\begin{array}{r}<0.001 \\
0.002\end{array}$ & 0.30 & 770.9 \\
\hline $\begin{array}{l}\text { GAMM 1: all species } \\
\text { s(dbh) } \\
\text { s(Neighborhood) } \\
\text { s(FPC) } \\
\text { Nitrogen } \\
\text { Species: } \text { E. ovata } \\
\text { Nitrogen } \times \text { species: E. ovata }\end{array}$ & $\begin{array}{r}0.06 \\
1.98 \\
-0.15\end{array}$ & $\begin{array}{r}144.0 \\
6.0 \\
5.4\end{array}$ & $\begin{array}{r}3.013 \\
2.392 \\
-2.808\end{array}$ & $\begin{array}{r}<0.001 \\
<0.001 \\
0.003 \\
0.003 \\
0.017 \\
0.005\end{array}$ & 0.31 & 5006.0 \\
\hline
\end{tabular}

Notes: Smoothed terms, denoted "s(...)," are illustrated in Fig. 3. "Neighborhood" describes the mean predicted number of koala visits to neighboring trees within a $25-\mathrm{m}$ radius. FPC indicates the concentration of formylated phloroglucinol compounds (lipid-soluble phenolics), potent herbivore deterrents in foliage of the tree.

$\uparrow$ For smooth terms, $z$ statistics apply to GAMs, and $F$ statistics apply to the GAMM.

$\$$ For parametric terms, the $\chi^{2}$ statistic applies to GAM 5 for nitrogen; $t$ statistics apply to the GAMM.

species or to predict palatability of E. ovata were unsuccessful.

The set of koala feeding observations used to develop the calibration for E. globulus (the "calibration set") included spectra from all trees except those identified as spectral outliers (Shenk and Westerhaus 1991). For E. viminalis, however, calibration sets including leaf samples from feeding trials in all experimental periods produced markedly less powerful predictive equations than those produced from any of the three groups separately. The final E. viminalis calibration set excluded the 1998 data but included 18 trees each from 2001 and 2003.

Among remnant trees of both species, the foliage of larger trees tended to be less palatable (based on dry matter intake, DMI) than that of smaller trees (linear regressions: for E. globulus, DMI = 207.2-0.37(dbh); $F_{1,374}=22.14, P<0.001, r^{2}=0.053$; for $E$. viminalis, $\mathrm{DMI}=170.2-0.54(\mathrm{dbh}) ; F_{1,374}=6.42, P=0.01, r^{2}=$ $0.033)$. A multiple linear regression $\left(F_{2,377}=67.8, P<\right.$ 0.001 ) explaining $26 \%$ of variation in predicted palatability for E. viminalis included as significant independent variables both FPC concentration $($ effect $=-4.45, P$
$<0.001)$ and nitrogen (effect $=2.77, P=0.04)$. An equivalent regression for E. globulus explained $24 \%$ of variation $\left(F_{2,492}=77.2, P<0.001 ; \mathrm{FPC}\right.$ effect $=-3.27$, $P<0.001 ;$ nitrogen effect $=2.66, P=0.007)$.

\section{Koala tree use}

Koalas were observed 895, 259, and 421 times in $E$. globulus, E. ovata, and E. viminalis, respectively. Neighborhood quality and dbh were highly significant predictors of koala visits to both E. globulus and $E$. viminalis (Table 1). Below about $60 \mathrm{~cm}$ dbh, the larger the tree, the more likely it was to be visited by koalas, but above $60 \mathrm{~cm}$, the likelihood of being visited was not related to dbh (Fig. 3).

For E. globulus, koala visits were positively associated with palatability (GAM 2; Table 1, Fig. 3). The explanatory power of this model was very similar to an alternative model (GAM 1) that included FPC concentration (negative association) and nitrogen (positive association); koala visits decreased below a nitrogen threshold of $12 \mathrm{mg} / \mathrm{g}$ DM (Table 1, Fig. 3). Similarly for E. viminalis, GAM 4 showed a positive association between koala visits and palatability and was equally 


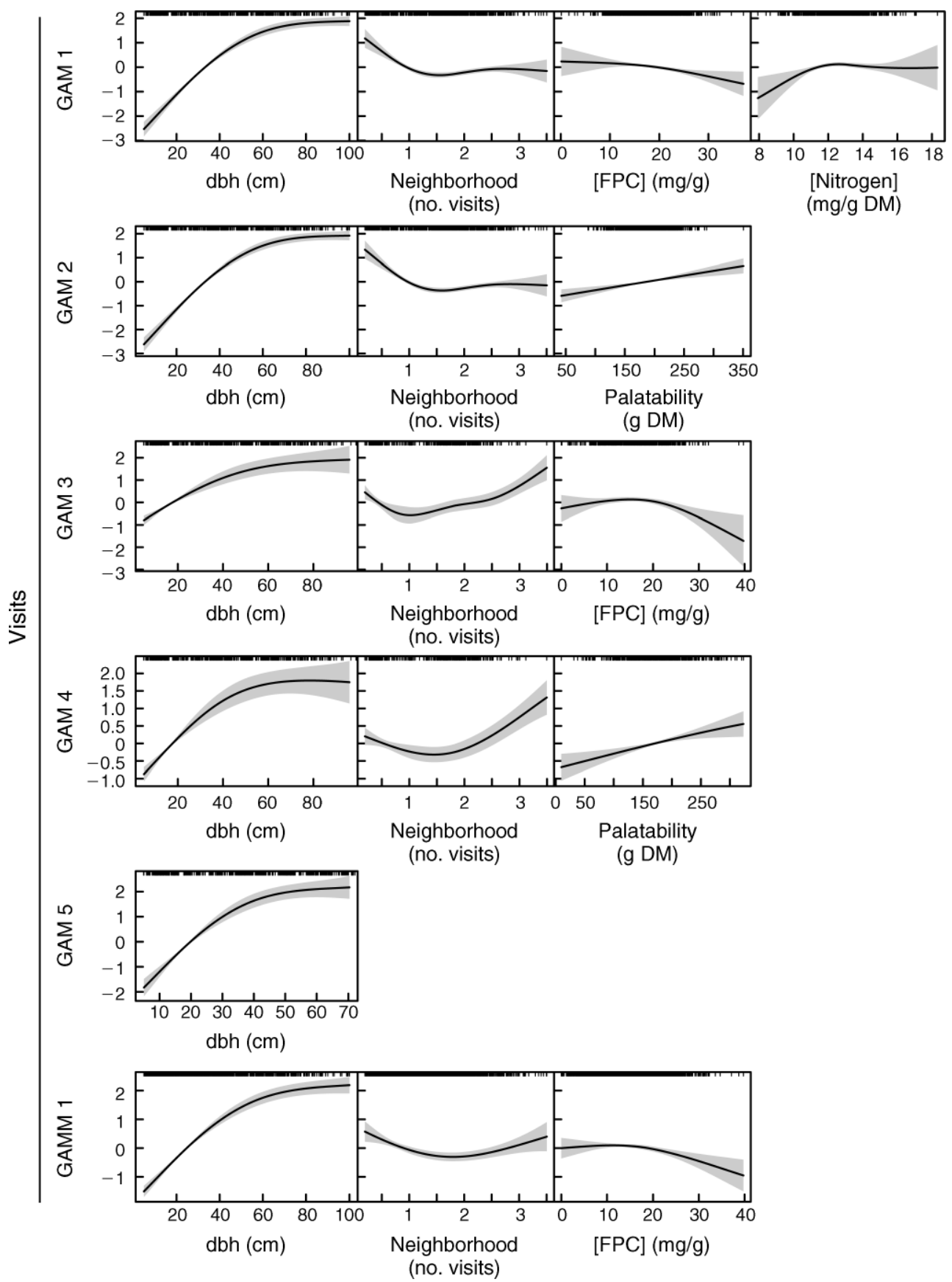

FIG. 3. Smooth terms included in generalized additive models (GAM) 1 and 2 (E. globulus), 3 and 4 (E. viminalis), and 5 (E. ovata) and generalized additive mixed model (GAMM) 1 (all species), described in Results: Koala tree use and in Table 1. Each row corresponds to one model; shaded regions show the $95 \%$ confidence interval around the smooth terms. These terms describe the relative likelihood of a tree being visited by a koala across the observed ranges of variation in tree size ("dbh," trunk diameter in cm at $130 \mathrm{~cm}$ height), mean predicted number of visits to neighboring trees in a 25 -m radius ("neighborhood"), foliar concentrations in $\mathrm{mg} / \mathrm{g} \mathrm{DM}$ of formylated phloroglucinol compounds that deter herbivores (FPC), and of nitrogen concentration and palatability (g DM eaten overnight) of foliage predicted using a near-infrared spectroscopic calibration based upon observations of feeding by captive koalas.

satisfactory as GAM 3, which showed that koala visits decreased as foliar FPC concentration increased above $\sim 20 \mathrm{mg} / \mathrm{g}$ DM. GAM 5 showed koala visits to $E$. ovata were explained by a positive relationship with dbh and, in this case, also by a negative relationship with nitrogen concentration (Table 1, Fig. 3).

Because the NIRS calibration for palatability of $E$. ovata was unsuccessful, it was not possible to include 

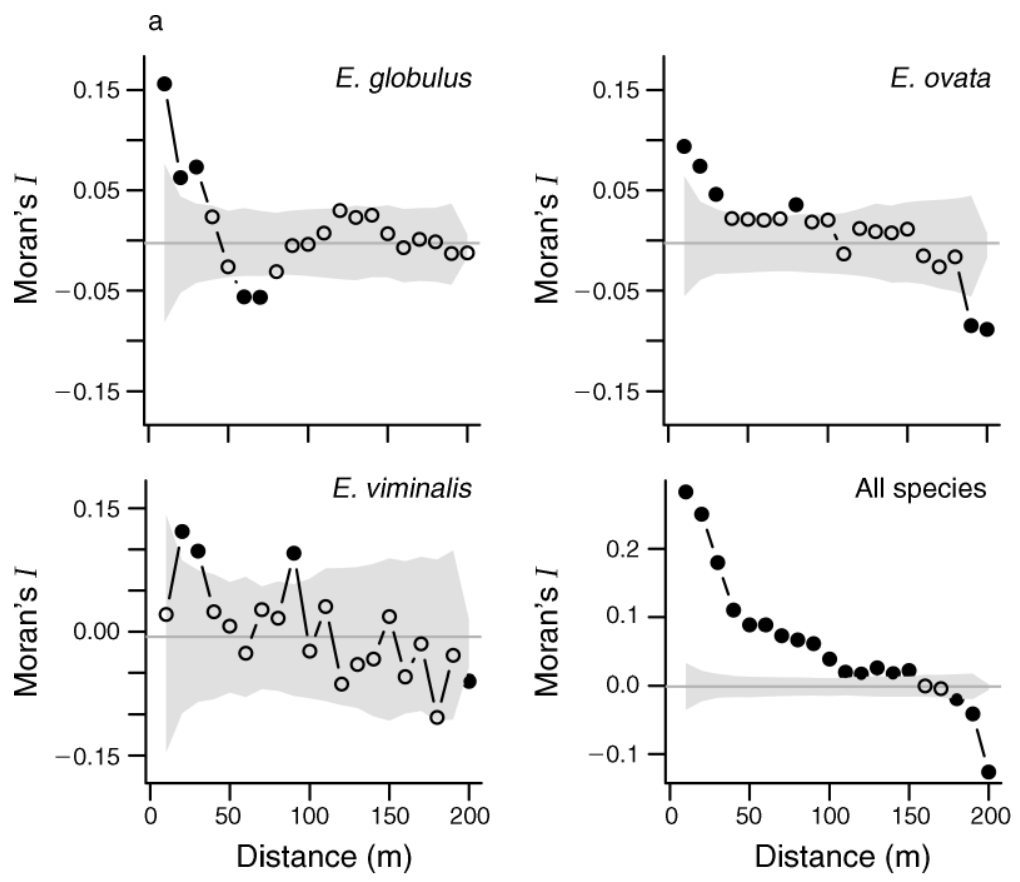

b) Koala movements

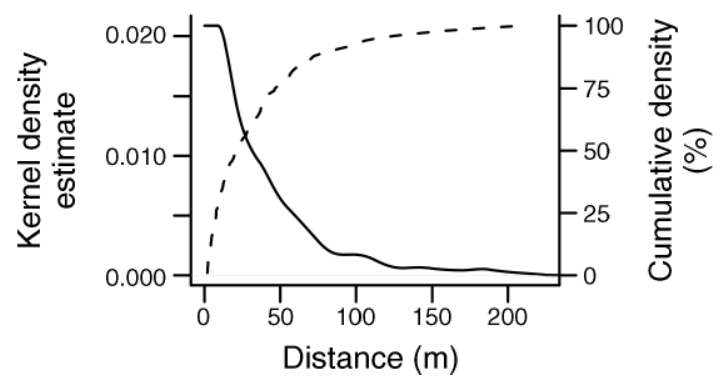

FIG. 4. (a) Autocorrelograms showing spatial autocorrelation (Moran's $I$ ) of FPC concentrations at 10-m intervals in $E$. globulus, E. ovata, E. viminalis, and all species together. $I$ was calculated across all tree pairs in each between-tree distance class (0-10 m, 10-20 m, and so forth). Shaded regions indicate a $95 \%$ confidence interval determined from 1000 permutations; solid circles indicate values that differ significantly $(P \leq 0.05)$ from the values of $I$ that would be expected, given a lack of autocorrelation (indicated by the gray horizontal line); open circles indicate nonsignificant values of $I$. (b) Gaussian kernel density estimate (solid line, left axis) and corresponding cumulative density function (dashed line, right axis) of observed tree-to-tree movements by female koalas at Phillip Island $(n=332)$. For the kernel density estimate, the estimated density at distances of less than $9 \mathrm{~m}$ was increased to match the maximum density value.

palatability in a GAMM describing visits to all three species. GAMM 1 (Table 1) reveals that after accounting for spatially autocorrelated residual error, koala visits were explained by dbh, neighborhood quality, FPC concentration, foliar nitrogen, and tree species $(E$. ovata or other). Koala visits were positively associated with nitrogen for E. viminalis and E. globulus, but negatively associated for E. ovata. The smoothed term for neighborhood quality was U-shaped, indicating that a tree's likelihood of koala visitation increased both in good neighborhoods (where neighboring trees were predicted to receive many koala visits) and in bad ones (where the opposite was true) compared to an average neighborhood (Fig. 3). GAMM 1 predicted that koala visits varied across a range of dbh, FPC concentrations, and neighborhood quality values (Fig. 3).

\section{Spatial autocorrelation}

FPC concentrations exhibited spatial autocorrelation up to a distance of $30 \mathrm{~m}$ in each species (Fig. 4a), and patchy species distributions (Fig. 1) and between-species differences in foliar chemistry (Moore et al. 2004b) produced much stronger spatial autocorrelation at distances of up to $150 \mathrm{~m}$ when all trees in the reserve were considered (Fig. 4a). In contrast to FPC concentrations, spatial autocorrelation in NIRS-predicted palatability for E. globulus and E. viminalis and in foliar nitrogen for all three species was insignificant. 
a) E. globulus tree locations, dbh, and [FPC]

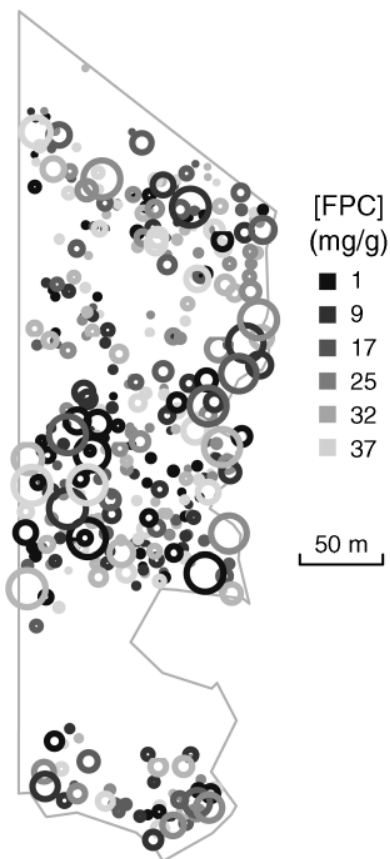

b) E. globulus FPC

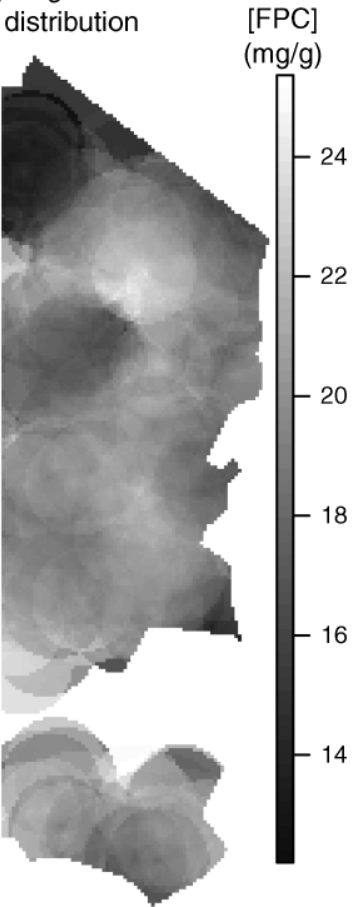

c) Predicted koala density (GAMM)

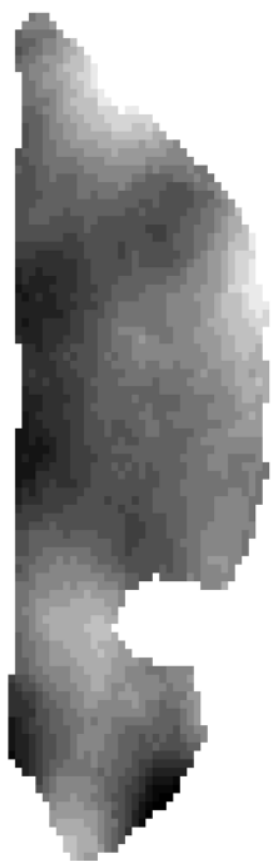

d) Observed koala density

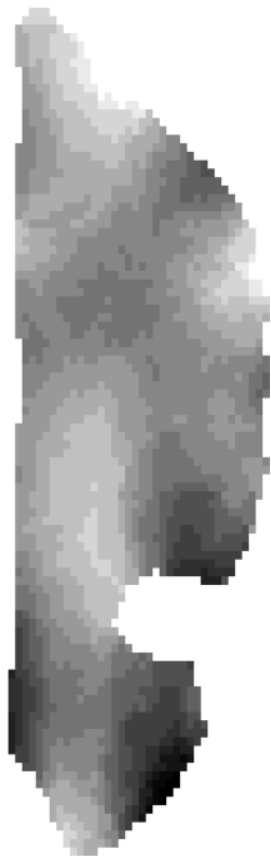

FIG. 5. (a) Map of remnant E. globulus trees. Size of the circles shows the relative dbh of trees, and the shade of gray indicates foliar FPC concentrations as indicated in the key. The gray outline delimits the extent of remnant woodland. (b) Interpolated surface generated using Eq. 1, illustrating mean FPC concentrations (gradient key shows $\mathrm{mg} / \mathrm{g}$ ) of E. globulus in a search radius of $30 \mathrm{~m}$ with a power constant of 0.4 . (c, d) Koala density maps generated using Eq. 2 with a maximum search radius $(\tau)$ of $50 \mathrm{~m}$ (c) from the predictions of GAMM 1 and (d) from observed koala tree use; shading indicates relative koala densities from high (dark shading) to low (light). The density scales are unitless; different points are weighted differently within each search radius, and some koalas were counted many times, at least a month apart. In panel (c), there is a 3.8 -fold difference across the range of minimum to maximum koala densities, with a 1.25 -fold difference across the interquartile range. For the observed koala surface in panel (d), the minimum and maximum densities show a 12-fold difference, with a 1.45 -fold difference across the interquartile range. The extensive variation in koala densities observed across the reserve was greater than that predicted.

\section{Mapping}

Of female koala tree-tree movements, $60 \%$ were between trees less than $30 \mathrm{~m}$ apart (Fig. 4b). An interpolated surface generated using Eq. 1, where $\tau=$ $30 \mathrm{~m}$ and $b=0.4$ highlighted several hotspots of highFPC phenotypes in the population of E. globulus (Fig. $5 b)$. A comparison of two interpolated surfaces from Eq. 2 ( $\tau=50 \mathrm{~m}$ and $k=$ the kernel shown in Fig. $4 \mathrm{~b}$ ) showed that the density of koala visits predicted by GAMM1 (Fig. 5c) correctly identified several local koala hotspots and cold spots in the density of observed koala visits (Fig. 5d). However, the two maps were less congruent along much of the left-hand side of the mapped area.

\section{DisCUSSION}

We have demonstrated for the first time that NIRS predictions of plant palatability derived from feeding behavior of captive animals describe an important individual plant trait that influences foraging by wild herbivores. This technique offers a novel, herbivoredriven perspective of food and habitat quality. We successfully developed NIRS models to predict DMI of foliage by koalas for two widely used koala food species.
The significant relationship between predictions made by these models and FPC and nitrogen concentrations is consistent with previous understandings of the factors influencing koala feeding preferences. The fact that the final calibrations were influenced both by positive and negative aspects of plant quality is remarkable, particularly given the fact that statistical analysis of the feeding experiments in isolation failed to detect a relationship between nitrogen and koala feeding (Moore et al. 2005).

A great strength of the generalized additive modeling approach adopted here is that splines can be inspected to reveal the true shape of the relationship between koala visits and each significant tree attribute. For example, smooth terms fitted in GAM 1 and 3 (Fig. 3) reveal threshold effects of nitrogen and FPC concentrations, respectively. Koalas apparently do not discriminate among E. globulus on the basis of foliar nitrogen as long as it exceeds a threshold (about $12 \mathrm{mg} / \mathrm{g}$ ); below that threshold, trees can escape koala herbivory to varying degrees: the lower the foliar nitrogen, the less likely koalas are to visit. 
Unexpectedly, one model suggested that koalas preferentially used $E$. ovata trees with lower, rather than higher, nitrogen concentrations. Total nitrogen is a crude index of protein availability to herbivores because protein that becomes bound to tannins is largely indigestible (DeGabriel et al. 2008), so a measure of digestible nitrogen might show a different relationship to koala tree use. It is also conceivable that an unmeasured leaf attribute, such as the cyanogenic glycoside prunasin, which has previously been reported from $E$. ovata (Gleadow et al. 2008), covaries with nitrogen concentrations and deters koalas.

The models also revealed that trees are more likely to be visited by koalas if they are growing in either a particularly poor neighborhood or a particularly good neighborhood, where the quality of the neighborhood is influenced by both the size of neighboring trees and their foliar chemistry. The observed effect is presumably the result of two processes operating simultaneously. The associational susceptibility of trees in particularly poor neighborhoods can be explained by an attractant-decoy effect (Milchunas and Noy-Meir 2002), whereby the trees attract koalas because alternatives in the immediate vicinity are poor by comparison. The associational susceptibility of trees in high-quality neighborhoods can be explained if koalas also make foraging decisions at a larger "patch" scale resulting in a preference for "good" neighborhoods. Other studies have also shown that herbivores make simultaneous foraging decisions at multiple scales in experimental arenas (Hjältén et al. 1993, Bergman et al. 2005), but the success of this study in describing these processes in natural, continuous vegetation illustrates the value of this fine-scale mapping approach.

The strong neighborhood effect on tree use by koalas emphasizes the importance of "seeing the forest for the trees" when considering spatial variation in habitat quality, because although trees themselves are variable and represent habitat patches, further larger-scale patchiness in their value to herbivores is also present. Fine-scale spatial autocorrelation was observed among FPC concentrations of each species considered. FPC concentrations are highly heritable in Eucalyptus (Andrew et al. 2005), and Andrew et al. (2007) showed in $E$. melliodora that strong spatial autocorrelation in FPC concentrations overlaid a highly congruent spatial structure in genetic variance. The strength and scale of autocorrelation observed in this study for FPCs are comparable to those in E. melliodora and those for genetic markers in Tasmanian E. globulus (Skabo et al. 1998), and are probably largely a product of isolation by distance (Heywood 1991). In addition to endogenous genetic factors, however, it is also conceivable that exogenous factors have contributed to the observed spatial structure, either through geographical heterogeneity in natural selection (Endler 1977, Linhart and Grant 1996) or through environmental influences on phenotypes (Gram and Sork 2001, Brenes-Arguedas and Coley 2005).

Mapping approaches such as the smoothed phenotypic surfaces presented here (Fig. 5) allow the identification of phenotypic hotspots, where the intensity of the trees' interactions with their herbivores may differ from elsewhere. This map, along with the analysis of spatial autocorrelation, reveals that the scale of patchiness in FPC concentrations is the same scale at which koalas forage, as half of all observed movements by female koalas in the reserve were of $30 \mathrm{~m}$ or less. As a consequence, after many movements from tree to tree, koalas will find themselves in trees of quality similar to the tree they have just left. For less mobile folivores, possibly including the common ringtail possums (Pseudocheirus peregrinus) in the reserve, which are far more sensitive to variation in FPC concentrations (Lawler et al. 1998, Moore et al. 2004a), and for insects, this scale of spatial structure might be a defining feature not only of the foraging landscape but also of fine-scale species distribution patterns. Furthermore, although the study site at Phillip Island was relatively small, flat, and edaphically homogenous, across larger and more diverse areas, greater intraspecific variation in the quality of trees can be expected (O'Reilly-Wapstra et al. 2002, Moore et al. 2004c), with correspondingly significant consequences for folivore habitat quality. These observations emphasize the importance of considering animal behavior and the scale of animal foraging decisions when assessing landscape condition for herbivores (Searle et al. 2007).

It is not surprising that tree size should so strongly affect where koalas are found during the day. Larger trees possess more foliage and thus represent larger feeding patches; however, they may also provide more shade and greater safety from perceived dangers on the ground. Although it has been demonstrated on Phillip Island that the presence of a koala in a tree is a reliable indicator that some feeding has occurred in the tree (Martin and Handasyde 1999; B. D. Moore and K. J. Marsh, unpublished data), these data are not ideal for investigating the effect of foliar quality on feeding. Koalas mostly feed at night, and particularly if koalas favor relatively smaller trees for feeding, then observed diurnal visits likely provide a biased snapshot of where feeding takes place. Isolated records of koala visits also ignore such subtleties as the variation in meal length and the number of meals eaten per tree visit that characterize koala feeding behavior (Marsh et al. 2007). These facts suggest that the models might somewhat overestimate the importance of tree size to koalas and underestimate the importance of foliar characteristics.

The map of predicted koala densities throughout the reserve is based upon model predictions of visits to individual trees, but the interpolation was directed by observed koala movement patterns. This map is one attempt to "scale up" the current understanding of habitat use at one of the smallest scales possible (the 
individual tree) to a larger scale. However, conservation professionals and landscape ecologists generally work at scales beyond the 7-ha reserve that we studied. For example, one previous multiscale model of koala habitat selection considered factors operating from the tree scale through the stand scale and patch scale to factors varying at a landscape scale of hundreds to thousands of hectares (McAlpine et al. 2008). Reynolds (2008) has commented that two major challenges in spatial ecology are to understand the effects of landscape heterogeneity on animal movement and to translate observations taken at small spatial and temporal scales into expected patterns at greater scales. More studies like this, that consider herbivory and associational effects in exhaustively documented, heterogeneous habitats, will help to address the first challenge; practical limitations to the extent at which habitats can be analyzed in such detail mean that ecologists need to develop new strategies to tackle the second challenge. In particular, they will need to design sampling strategies that trade off spatial scale against resolution but describe habitats in a way meaningful to herbivores, while remaining conscious that animals can change movement patterns over time and in different habitats (Morales and Ellner 2002).

Although this is the first demonstration that NIRS can be used to predict herbivore responses to plant quality directly from NIRS spectra, the principles are applicable to the majority of plant-herbivore interactions and the technique offers a promising route to rapidly describe plant and habitat quality at a variety of scales (Foley et al. 1998). Furthermore, remote-sensing techniques can already detect foliar biochemistry at the scale of individual trees and patches (Kokaly et al. 2009), making the development of high-resolution, large-scale maps a worthy goal for researchers in the future. It is our hope that the approaches presented here offer a useful way forward for ecologists as they attempt to understand the way in which physical, chemical, and spatial aspects of habitat ultimately interact to determine the presence or absence of individuals and populations of animals, as well as the susceptibility of plants to herbivores, at a variety of scales.

\section{ACKNOWLEDGMENTS}

For assistance with field and laboratory work, thanks to Miranda Ebbers, Robert Bednarik, Ashley Reed, Kath Handasyde, Marg Healy, Peter Dann, Ros Jessop, Karen Ford (née Marsh), Gavin Moore, Phillip Island Nature Park, and Friends of the Koalas, Inc. Thanks to Jane DeGabriel and Glenn Iason for helpful comments on the manuscript. All experiments were approved by the Animal Experimentation and Ethics Committees of the Australian National University (F.BTZ.67.97) and Phillip Island Nature Park (PINP 1.98 and 2.01). Funding was provided to W. J. Foley from the Australian Research Council.

\section{Literature Cited}

Andrew, R. L., R. Peakall, I. R. Wallis, and W. J. Foley. 2007. Spatial distribution of defense chemicals and markers and the maintenance of chemical variation. Ecology 88:716-728.
Andrew, R. L., R. Peakall, I. R. Wallis, J. T. Wood, E. J. Knight, and W. J. Foley. 2005. Marker-based quantitative genetics in the wild? The heritability and genetic correlation of chemical defences in Eucalyptus. Genetics 171:1989-1998.

Atsatt, P. R., and D. J. O'Dowd. 1976. Plant defense guilds. Science 193:24-29.

Barbosa, P., J. Hines, I. Kaplan, H. Martinson, A. Szczepaniac, and Z. Szendrei. 2009. Associational resistance and associational susceptibility: having right or wrong neighbors. Annual Review of Ecology, Evolution, and Systematics 40: $1-20$.

Barnes, R. J., M. S. Dhanoa, and S. J. Lister. 1989. Standard normal variate transformation and detrending of near infrared reflectance spectra. Applied Spectroscopy 43:772-777.

Bergman, M., G. R. Iason, and A. J. Hester. 2005. Feeding patterns by roe deer and rabbits on pine, willow and birch in relation to spatial arrangement. Oikos 109:513-520.

Bergvall, U. A., P. Rautio, K. Kesti, J. Tuomi, and O. Leimar. 2006. Associational effects of plant defences in relation to within- and between-patch food choice by a mammalian herbivore: neighbor contrast susceptibility and defence. Oecologia 147:253-260.

Brenes-Arguedas, T., and P. D. Coley. 2005. Phenotypic variation and spatial structure of secondary chemistry in a natural population of a tropical tree species. Oikos 108:410 420.

Bryant, J. P., F. D. Provenza, J. Pastor, P. B. Reichardt, T. P. Clausen, and J. T. Dutoit. 1991. Interactions between woody plants and browsing mammals mediated by secondary metabolites. Annual Review of Ecology and Systematics 22: 431-446.

Bryant, J. P., G. D. Wieland, P. B. Reichardt, V. E. Lewis, and M. C. McCarthy. 1983. Pinosylvin methyl ether deters snowshoe hare feeding on green alder. Science 222:10231025.

Covelo, F., and A. Gallardo. 2004. Green and senescent leaf phenolics showed spatial autocorrelation in a Quercus robur population in northwestern Spain. Plant and Soil 259:267276.

DeGabriel, J. L., I. R. Wallis, B. D. Moore, and W. J. Foley. 2008. A simple, integrative assay to quantify nutritional quality of browses for herbivores. Oecologia 156:107-116.

Endler, J. A. 1977. Geographic variation, speciation, and clines. Princeton University Press, Princeton, New Jersey, USA.

Feng, Z. L., R. S. Liu, D. L. DeAngelis, J. P. Bryant, K. Kielland, F. S. Chapin, and R. Swihart. 2009. Plant toxicity, adaptive herbivory, and plant community dynamics. Ecosystems 12:534-547.

Foley, W. J., A. McIlwee, I. Lawler, L. Aragones, A. P. Woolnough, and N. Berding. 1998. Ecological applications of near infrared reflectance spectroscopy: a tool for rapid, cost-effective prediction of the composition of plant and animal tissues and aspects of animal performance. Oecologia 116:293-305.

Gleadow, R. M., J. Haburjak, J. E. Dunn, M. E. Conn, and E. E. Conn. 2008. Frequency and distribution of cyanogenic glycosides in Eucalyptus L'Hérit. Phytochemistry 69:18701874.

Gram, W. K., and V. L. Sork. 2001. Association between environmental and genetic heterogeneity in forest tree populations. Ecology 82:2012-2021.

Heywood, J. S. 1991. Spatial analysis of genetic variation in plant populations. Annual Review of Ecology and Systematics 22:335-355.

Hjältén, J., K. Danell, and L. Ericson. 2004. Hare and vole browsing preferences during winter. Acta Theriologica 49: $53-62$.

Hjältén, J., K. Danell, and P. Lundberg. 1993. Herbivore avoidance by association: vole and hare utilization of woody plants. Oikos 68:125-131. 
Kokaly, R. F., G. P. Asner, S. V. Ollinger, M. E. Martin, and C. A. Wessman. 2009. Characterizing canopy biochemistry from imaging spectroscopy and its application to ecosystem studies. Remote Sensing of Environment 113:S78-S91.

Lawler, I. R., W. J. Foley, and B. M. Eschler. 2000. Foliar concentration of a single toxin creates habitat patchiness for a marsupial folivore. Ecology 81:1327-1338.

Lawler, I. R., W. J. Foley, B. M. Eschler, D. M. Pass, and K. Handasyde. 1998. Intraspecific variation in Eucalyptus secondary metabolites determines food intake by folivorous marsupials. Oecologia 116:160-169.

Lawler, I. R., J. Stapley, W. J. Foley, and B. M. Eschler. 1999. Ecological example of conditioned flavor aversion in plantherbivore interactions: Effect of terpenes of Eucalyptus leaves on feeding by common ringtail and brushtail possums. Journal of Chemical Ecology 25:401-415.

Lennon, J. J. 2000. Red-shifts and red herrings in geographical ecology. Ecography 23:101-113.

Linhart, Y. B., and M. C. Grant. 1996. Evolutionary significance of local genetic differentiation in plants. Annual Review of Ecology and Systematics 27:237-277.

Liu, H., and K. S. Chan. 2008. Constrained generalized additive mixed model with zero-inflated data. Technical Report 388. Department of Statistics and Actuarial Science, University of Iowa, Iowa City, Iowa, USA.

Marsh, K. J., I. R. Wallis, and W. J. Foley. 2007. Behavioural contributions to the regulated intake of plant secondary metabolites in koalas. Oecologia 154:283-290.

Martin, R. W., and K. A. Handasyde. 1999. The koala: natural history, conservation and management. Second edition. University of New South Wales Press, Sydney, Australia.

McAlpine, C. A., J. R. Rhodes, M. E. Bowen, D. Lunney, J. G. Callaghan, D. L. Mitchell, and H. P. Possingham. 2008. Can multi-scale models of a species' distribution be generalised from region to region? A case study of the koala. Journal of Applied Ecology 45:558-567.

McIlwee, A. M., I. R. Lawler, S. J. Cork, and W. J. Foley. 2001. Coping with chemical complexity in mammal-plant interactions: near-infrared spectroscopy as a predictor of Eucalyptus foliar nutrients and of the feeding rates of folivorous marsupials. Oecologia 128:539-548.

Milchunas, D. G., and I. Noy-Meir. 2002. Grazing refuges, external avoidance of herbivory and plant diversity. Oikos 99:113-130.

Moore, B. D., and W. J. Foley. 2000. A review of feeding and diet selection in koalas (Phascolarctos cinereus). Australian Journal of Zoology 48:317-333.

Moore, B. D., and W. J. Foley. 2005. Tree use by koalas in a chemically complex landscape. Nature 435:488-490.

Moore, B. D., W. J. Foley, I. R. Wallis, A. Cowling, and K. A. Handasyde. 2005. Eucalyptus foliar chemistry explains koala feeding preferences. Biology Letters 1:64-67.

Moore, B. D., I. R. Wallis, K. J. Marsh, and W. J. Foley. 2004a. The role of nutrition in the conservation of the marsupial folivores of eucalypt forests. Pages 549-575 in D. Lunney, editor. Conservation of Australia's forest fauna. Royal Zoological Society of New South Wales, Mosman, NSW, Australia.

Moore, B. D., I. R. Wallis, J. Palá Paúl, J. J. Brophy, R. H. Willis, and W. J. Foley. 2004b. Anti-herbivore chemistry of Eucalyptus: cues and deterrents for marsupial folivores. Journal of Chemical Ecology 30:1743-1769.

Moore, B. D., I. R. Wallis, J. Wood, and W. J. Foley. 2004c. Foliar nutrition, site quality and temperature affect foliar chemistry of tallowwood (Eucalyptus microcorys). Ecological Monographs 74:553-568.

Morales, J. M., and S. P. Ellner. 2002. Scaling up animal movements in heterogeneous landscapes: the importance of behaviour. Ecology 83:2240-2247.

O'Reilly-Wapstra, J. M., C. McArthur, and B. M. Potts. 2002. Genetic variation in resistance of Eucalyptus globulus to marsupial browsers. Oecologia 130:289-296.

Provenza, F. D., J. J. Villalba, C. D. Cheney, and S. J. Werner. 1998. Self-organization of foraging behavior: From simplicity to complexity without goals. Nutrition Research Reviews 11: 199-222.

Reynolds, A. 2008. How many animals really do the Lévy walk? Ecology 89:2347-2351.

Schreiber, L. A., and R. K. Swihart. 2009. Selective feeding of pine voles on roots of tree seedlings. Canadian Journal of Zoology 87:183-187.

Searle, K. R., N. T. Hobbs, and I. J. Gordon. 2007. It's the "foodscape", not the landscape: using foraging behavior to make functional assessments of landscape condition. Israel Journal of Ecology and Evolution 53:297-316.

Shenk, J. S., and M. O. Westerhaus. 1991. Population definition, sample selection and calibration procedures for near infrared reflectance spectroscopy. Crop Science 31:469474.

Skabo, S., R. E. Vaillancourt, and B. M. Potts. 1998. Fine-scale genetic structure of Eucalyptus globulus ssp. globulus forest revealed by RAPDs. Australian Journal of Botany 46:583594.

Stolter, C., R. Julkunen-Tiitto, and J. U. Ganzhorn. 2006. Application of near infrared reflectance spectroscopy (NIRS) to assess some properties of a sub-arctic ecosystem. Basic and Applied Ecology 7:167-187.

Wallis, I. R., and W. J. Foley. 2003. Validation of near-infrared reflectance spectroscopy to estimate the potential intake of Eucalyptus foliage by folivorous marsupials. Australian Journal of Zoology 51:95-98.

Wood, S. N. 2006. Generalized additive models: an introduction with R. Chapman and Hall/CRC, Boca Raton, Florida, USA.

Wood, S. N. 2008. Fast stable direct fitting and smoothness selection for generalized additive models. Journal of the Royal Statistical Society B 70:495-518.

\section{APPENDIX}

Details of calibration sets, treatment of spectra, and regression statistics for NIRS modified partial least-squares regression models predicting dry matter intake (in grams) of foliage of Eucalyptus globulus and E. viminalis by koalas (Ecological Archives E091-222-A1). 